\title{
Exploring the Gain of Opportunistic Routing in Wireless Multicast
}

\author{
Tan Le and Yong Liu \\ Department of Electrical and Computer Engineering \\ Polytechnic Institute of NYU \\ New York, NY 11201 \\ Email: tle04@students.poly.edu, yongliu@poly.edu
}

\begin{abstract}
Opportunistic Routing (OR) has recently been proposed to improve the efficiency of unicast in multi-hop wireless networks. OR exploits the broadcast nature of wireless transmission medium and opportunistically selects a relay path to deliver a packet to its receiver. In this paper, we explore the gain of adopting $O R$ in wireless multicast. The main challenge is to efficiently share opportunistic relay paths between multiple receivers. We first propose a scheme using Minimum Steiner Tree with Opportunistic Routing (MSTOR). The source and receivers are connected by a Steiner tree. Packets are transmitted using Unicast OR on each link of the tree. MSTOR does not exploit opportunistic receptions cross different links in the tree. We then propose a Multicast OR (MOR) algorithm. Starting from the source, MOR opportunistically employs a set of forwarders to push a packet closer to all receivers round-by-round. Each forwarder is responsible for sending the packet to a subset of receivers, and transmits the packet once each round. Based on packet receptions at the end of each round, a new forwarder set is constructed to maximize the expect transmission advancement towards all receivers. We study the performance improvement of MSTOR and MOR using numerical simulations. Our results demonstrate that both MOR and MSTOR can achieve a much higher multicast efficiency than the original Unicast $O R$ and the traditional minimum multicast-tree based schemes. MOR constantly achieves significant transmission cost savings in all studied cases.
\end{abstract}

\section{INTRODUCTION}

The increasing popularity of wireless devices and new wireless applications make it important to delivery multicast services efficiently over multi-hop wireless networks. It is well-known that the general minimum-cost multicast routing problem is NP-hard. Wireless multicast additionally has to deal with interferences and collisions on volatile wireless links. While some attempts [1][2][3][4][5] have been made to achieve a high efficiency in wireless multicast, the research field is still largely open. In parallel, Opportunistic Routing (OR) [6][7][8][9][10][11] has recently been proposed to improve the efficiency of unicast in multi-hop wireless networks. OR exploits the broadcast nature of wireless transmission medium and opportunistically selects a relay path to deliver a packet to its receiver. By exploiting opportunistic packet receptions, OR can significantly reduce the number transmissions necessary to deliver a packet to its receiver. It is therefore tempting to adopt OR to improve the efficiency of wireless multicast. However, the adoption of OR in multicast is not straightforward. The main challenge is how to efficiently share opportunistic relay paths between multiple receivers. To illustrate, let's look at a toy example presented in Figure 1. A source node $S$ needs to multicast one packet

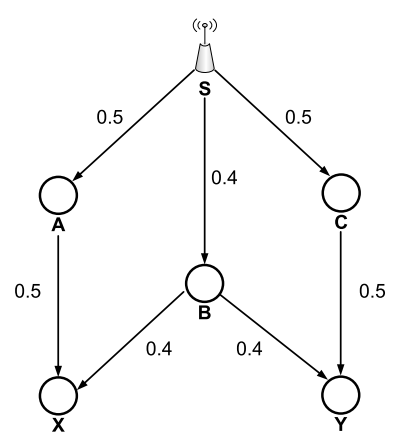

Fig. 1. Multicast Schemes in a Multi-hop Wireless Network

to receivers $X$ and $Y$. The Packet Reception Ratio (PRR) on wireless links are shown in the Figure. With traditional multicast routing, the packet can be sent along the multicast tree $S \Rightarrow\langle B\rangle \Rightarrow\langle X, Y\rangle$. Multicast tree approach does not exploit the the opportunistic receptions on $A$ and $C$ when $S$ sends the packet to $B$. To achieve the OR gain, $S$ can conduct two Unicast OR to send a packet to $X$ and $Y$ separately. Since nodes $A$ and $B$ are closer to $X$ than $S, S$ will choose $\{A, B\}$ as the candidate relay set to forward the packet to $X$. Since $A$ has a more reliable link to $X$, it will get higher priority than $B$ in the relay set. If both $A$ and $B$ get the packet transmitted by $S, A$ will take over and relay the packet to $X$. Likewise, $\{B, C\}$ is $S$ 's candidate relay set for $Y$, and $C$ has higher relay priority than $B$. The expected number of transmissions is two times that of individual Unicast OR. To combine the opportunistic relay paths to $X$ and $Y, S$ can broadcast the packet to the relay set $\{A, B, C\}$. If $B$ receives the packet, it will send the packet to $X$ and $Y$ through one broadcast. If $B$ does not received the packet but $A$ and $C$ receives it successfully, $A$ will relay the packet to $X$ and $C$ will relay the packet to $Y$. In all other cases, $S$ will retransmit the packet. The last scheme combines the merits of multicast and OR and is more efficient than pure unicast OR and pure multicast tree approaches. However, multicast OR in general network 
topology is still an open challenging problem.

In this paper, we explore the gain of OR in wireless multicast by studying two schemes. The first scheme combines Minimum Steiner Tree with Unicast OR (MSTOR). We allow any pair of nodes in the network communicate using Unicast OR. The distance between two nodes is measured by the cost of Unicast OR between the two. Then we build a minimum Steiner tree to connect the source with all receivers. A packet from the source will be disseminated to all receivers along the tree, with Unicast OR employed on each link of the tree. MSTOR does not exploit opportunistic receptions cross different links in the tree. The second scheme, Multicast OR (MOR), extends the concept of OR directly to cover multiple receivers. Starting from the source, MOR opportunistically employs a set of forwarders to push a packet closer to all receivers round-by-round. Each forwarder is responsible for sending the packet to a subset of receivers, and transmits the packet once each round. Based on packet receptions at the end of each round, a new forwarder set is constructed to maximize the Expected Transmission Advancement towards all receivers. To the best of our knowledge, this is the first work on integrating OR with wireless multicast. We evaluate the performance improvement of MSTOR and MOR through numerical simulations in example networks. Our results demonstrate that both MOR and MSTOR can achieve a much higher multicast efficiency than the original Unicast OR and the traditional minimum multicast-tree based schemes. MOR constantly achieves significant transmission cost savings in all studied cases.

The rest of the paper is organized as follows. We briefly review the related work on wireless multicast and present OR concepts necessary for our proposed schemes in Section II. We formally describe the network model under study in Section III. The MSTOR scheme is studied in Section IV. We present the MOR scheme in Section V. The performance of MSTOR and MOR are evaluated through case studies on several example networks in Section VI. The paper is concluded in Section VII.

\section{RALATED WORK}

\section{A. Multicast Routing}

Wireless multicast routing have been investigated for a while since it is a more efficient method of supporting group communication than unicast or broadcast routing. There are many researchs on this important topic. In [1], the authors presents On-Demand Multicast Routing Protocol (ODMRP) for wireless mobile ad hoc networks, in which applies ondemand procedures to dynamically build routes and maintain multicast group membership. Authors of [2] develop the broadcast incremental power algorithm based on tree construction and adapt it to multicast operation to exploit the broadcast nature of the wireless communication environment. In [3], the authors find a heuristic communication strategy on wireless multicast network that minimizes the total deadlines missed across all receivers, where a receiver counts a miss if it does not receive a packet by its deadline. The work in [4] proposed the probabilistically reliable on-demand wireless multicast routing scheme base on Expected Multicast Transmissions metric, which gaining better performance than Shortest Path Tree and the Minimum Forwarder Tree mechanisms. In [5], the authors propose a simple comb-based architecture for multicast routing which achieves the same order-optimal results as the approach of constructing Steiner tree while requiring little location information and no computational overhead.

\section{B. Opportunistic Routing}

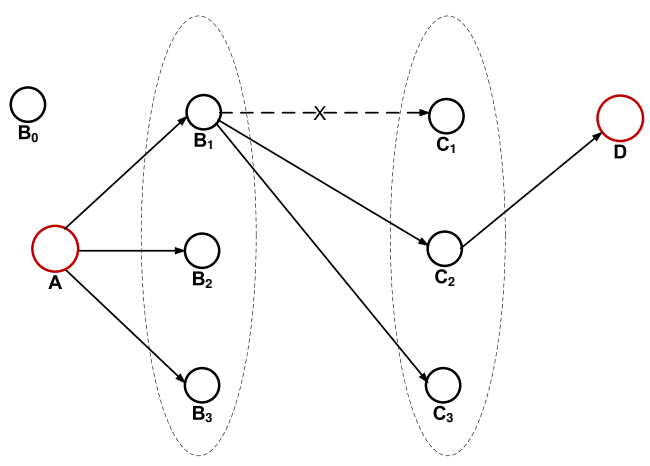

Fig. 2. Unicast Opportunistic Routing on ad-hoc network

Recently, the topic Opportunistic Routing on ad-hoc networks attracted lots of interest. In [6], the authors studied the opportunistic routing protocol ExOR, which dynamically chooses paths on a per-transmission basis in a wireless network to efficiently improve the throughput. To illustrate the idea of OR, in Figure 2, $A$ wants to send packets to D. $B_{1}$, $B_{2}$ and $B_{3}$ are closer to $D$ and are chosen as the candidate forwarders. After one broadcast from $A, B_{1}, B_{2}$, and $B_{3}$ all receive the packet. Assuming $B_{1}$ has the highest forwarding priority, so it will take over and broadcast the packet to its candidate forwarders $C_{1}, C_{2}$ and $C_{3}$. Assume highest priority node $C_{1}$ misses the packet, so $C_{2}$ will take over and forward the packet to its receiver $D$. For convenient to classify this OR routing scheme with the new one proposed in our paper, we call this schemse is "Unicast Opportunistic Routing" (Unicast OR).

In [7], the authors introduced the robust distribution opportunistic routing scheme base on ETX metric that can find the optimal path from source to receiver. Authors of [8] conducted a systematic performance evaluation, taking into account node densities, channel qualities and traffic rates to identify the cases when opportunistic routing makes sense. The work in [9] proposed the method to calculate the maximum throughput between two end nodes with Opportunistic Routing in ad-hoc networks. The recent work from [10] studies the capacity of hybrid wireless networks with opportunistic routing, which exploit high speed data transmissions in infrastructure network through base stations to improve the routing performance. The work with closed topic in [11] investigates the opportunistic routing in both unicast and multicast cases. This work deploys the advantages from network coding to improve performance of OR on wireless multicast network. 


\section{Optimal Unicast OR from Single Source to Single receiver}

The recent work from Laufer et al. [12] proposed the SAF and SMAF algorithms to calculate the optimal OR path between two nodes with single and multiple transmission rates. These schemes were proved to have the same complexity of Dijkstra's shortest path algorithm. For the single-rate case,

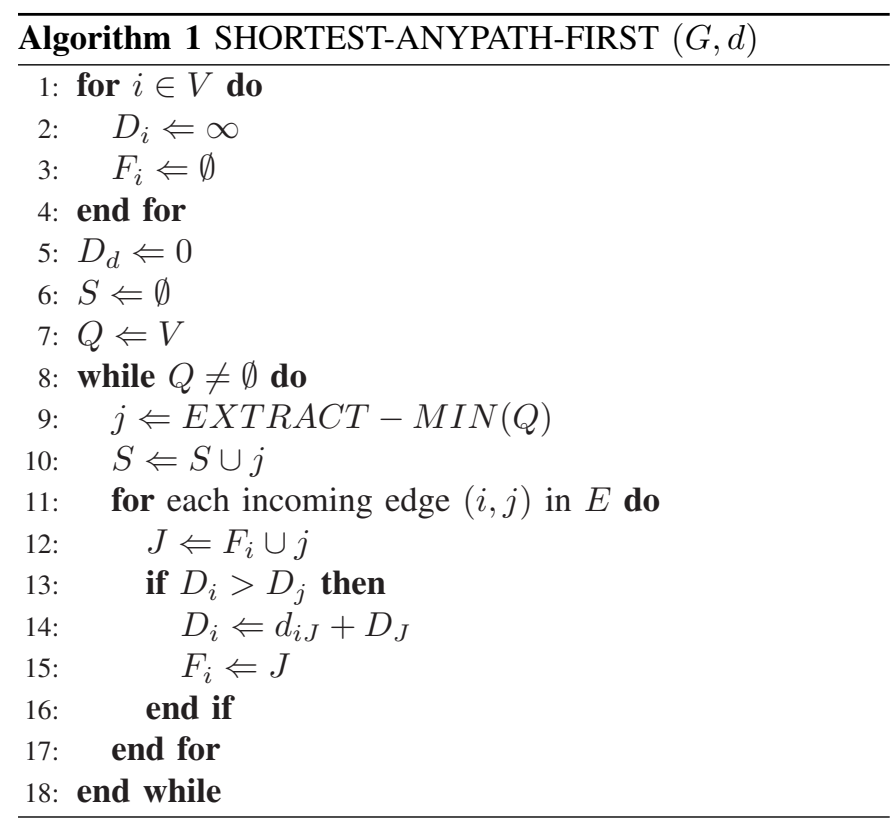

given a graph $G=(V, E)$, the Algorithm 1 calculates the shortest OR paths from every node in the network to a single receiver $d$. For every node $i \in V, D_{i}$ is the distance of the shortest OR path to $d . F_{i}$ is the candidate forwarding set for node $i . d_{i J}$ is the Anycast Link Cost from a transmitter node $i$ to a neighbor set $J$. That is the expected number of transmissions from $i$ to ensure at least one neighbor in $J$ get the packet.

$$
d_{i J}=\frac{1}{1-\prod_{j \in J}\left(1-p_{i j}\right)}
$$

where $p_{i j}$ is the Packet Reception Ratio (PRR) on the wireless link between $i$ and $j$. In set $J=\langle 1,2, \ldots, n\rangle$, nodes are sorted by their shortest OR path cost to the receivers, i.e., that $\left\langle D_{1}<\right.$ $\left.D_{2}<\ldots<D_{n}\right\rangle$. Assume $p_{i k}=0$ with $k=0$. Then the remaining path cost is:

$$
D_{J}=\frac{1}{1-\prod_{j \in J}\left(1-p_{i j}\right)}\left(\sum_{j=1}^{n} p_{i j} D_{j}\left(\prod_{k=0}^{j-1}\left(1-p_{i k}\right)\right)\right)
$$

The set $S$ stores the set of nodes for which we already found a shortest OR paths. We store each node $i \in V-S$ for which we still do not have a shortest OR path in a priority queue $Q$ keyed by their $D_{i}$ values. The complexity of the algorithm is $O(V \log V+E)$.

\section{Network Model And Assumptions}

We consider a network of $N$ static wireless nodes, including 1 source node $S, K<N$ receivers $D_{1}, D_{2}, \ldots, D_{K}$, and
$N-K-1$ relay nodes. All nodes are equipped with radio interfaces and can communicate with neighbor nodes within their transmission ranges. Wireless links between neighbor nodes are not reliable. The success probability of packet transmission on a link is given by the Packet Reception Ratio (PRR). The PRR $p_{i j}$ of link $\langle i, j\rangle$ theoretically depends on the distance between nodes $i$ and $j$, node density and traffic around $i$ and $j$, and the MAC scheduling scheme. Assume every PRR $p_{i j}$ of link $\langle i, j\rangle$ is independent with PRR of other links.

\section{Minimum Steiner Tree Opportunistic Routing}

Our first scheme is to combine OR with the minimum multicast tree (Steiner tree). In order to minimize the cost of multicast transmissions from a source to a set of receivers, we need to build up the minimum Steiner tree that connects the source with all receivers. The source and receivers are called terminal nodes, other wireless nodes of the minimum Steiner tree are called Steiner nodes. Ideally, the minimum Steiner tree is the mutlicast tree with the minimum total transmission cost. Given the transmission cost on each link, it is wellknown that finding minimum Steiner tree is NP-Hard. To make it worse, the transmission cost on a wireless link cannot be independently defined. The transmissions on adjacent wireless links interfere with each other. Broadcast from one node sends out multiple copies of a packet along multiple outgoing links of the node. Traditional Steiner tree algorithms developed for wireline networks cannot be copied into wireless networks. Several new multicast tree algorithms [4], [13] have been proposed for wireless networks. However, those algorithms didn't exploit the gain of OR. In this section, we propose a wireless multicast scheme using minimum Steiner tree with Unicast OR.

\section{A. Minimum Steiner Tree algorithm}

Assume any pair of nodes in the network can reach each other using Unicast OR. Every node $i$ got the shortest OR distance $L_{i j}$ to other node $j$ based on the SAF algorithm [12] presented in Section II-C. $L_{i j}$ is the expected cost of transmitting a packet from $i$ to $j$ using Unicast OR. We construct a fully connected virtual graph $G=(V, E)$, with $V$ consisting of all nodes in the network and $E=V \times V$. The cost of the virtual link between $i$ and $j$ is the shortest path OR distance $L_{i j}$ in the underlying wireless network. In the virtual graph, we construct the minimum Steiner tree connecting the source node $S$ with the set of receivers $\mathcal{D}$. The most popular algorithm to construct the minimum Steiner tree was proposed by Dreyfus and Wagner [14] based on dynamic programming. The complexity of the algorithm is $O *\left(3^{k}\right)$. However, the dynamic nature of wireless ad-hoc networks requires a simpler minimum Steiner tree algorithm. In the following, we propose a simple heuristic algorithm to calculate a Steiner tree for our purpose. Let $\operatorname{MST}(X)$ be the cost of the Minimum Spanning Tree of the set $X$. Several popular algorithms to calculate $\operatorname{MST}(X)$ takes very close to linear time. Let $Y=S \cup \mathcal{D}$ be the set of terminal nodes. The other nodes on the tree called 


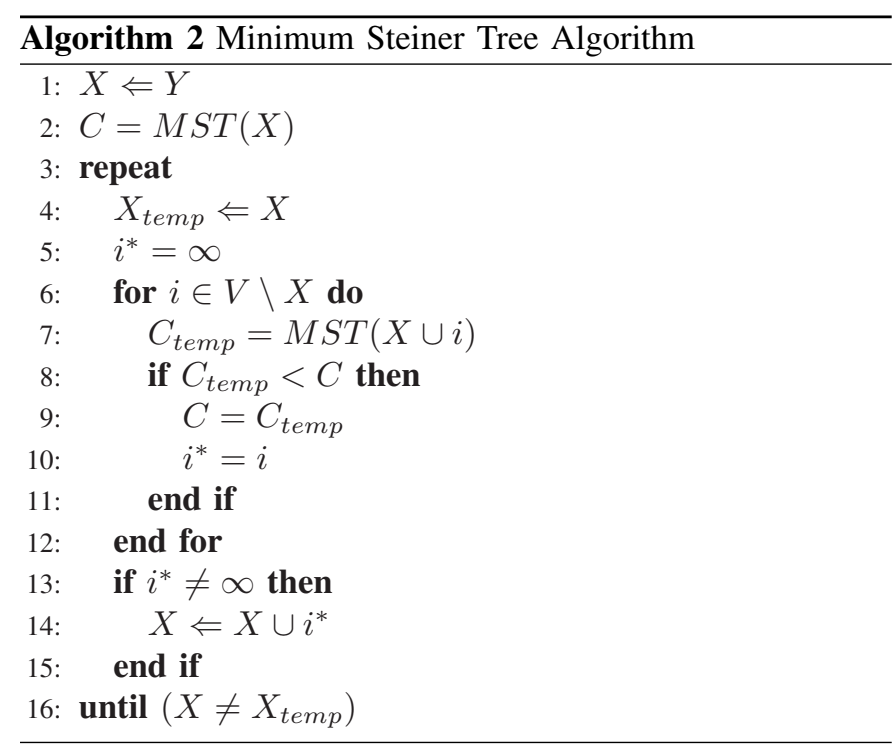

Steiner nodes. In the above algorithm, set $X$ stands for the set of nodes, that already admitted into the minimum Steiner tree. Variable $C$ stores the cost of the minimum Steiner tree. The idea is to first construct the Minimum Spanning Tree among all terminal nodes, then grow the the spanning tree into a Steiner tree by incorporating Steiner nodes step by step. At each step, the algorithm will find a node $i^{*}$, that when added to the tree could maximize the reduction of the total cost of the current tree. The algorithm completes when the cost could not be further improved. This simple algorithm take $O\left(V^{2} E\right)$ time to complete.

The obtained Steiner tree in the virtual graph will be mapped to a set of relay paths connecting the source with all receivers in the underlying wireless network. Each virtual link between two nodes is mapped to a Unicast OR relay path between the two nodes in the underlying wireless network. A packet will be transmitted using Unicast OR along all the relay paths until it reaches all the receivers. We call this scheme is "Minimum Steiner Tree opportunistic routing" - (MSTOR).

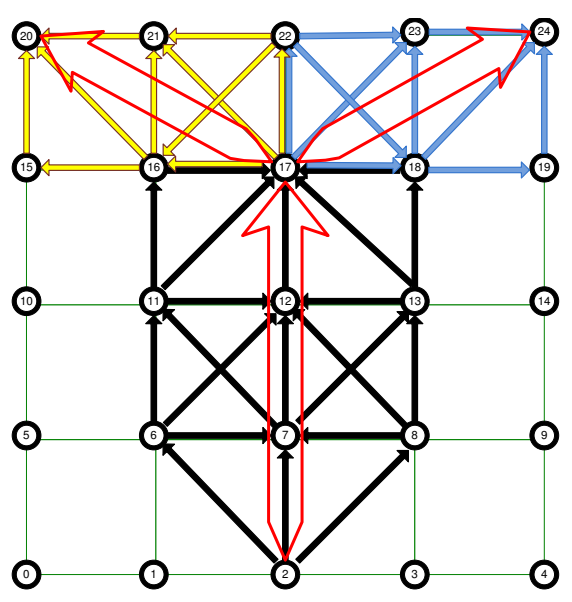

Fig. 3. Example of Minimum Steiner Tree OR algorithm

\section{B. Example of MSTOR and analysis}

Let's take a look at the following simple example in Figure 3. Assuming we have a grid network with grid size $100 \mathrm{~m} \mathrm{x}$ $100 \mathrm{~m} .25$ nodes are located on the site with homogeneous radio transmission range $150 \mathrm{~m}$. For demonstration, we use a simple Packet Reception Ratio (PRR) model on link $\langle i, j\rangle$ :

$$
p_{i j}=1-\frac{d_{i j}}{R},
$$

where $d_{i j}$ is the geographical distance between $i$ and $j$ and $R$ is the maximum radio transmission range. For the convenience of computation, assuming PRR on every link is independent with each other. Then the PRR of every wireless link on the network could be calculated. For example:

$$
\begin{gathered}
p_{12}=1-\frac{d_{12}}{R}=1-\frac{100}{150}=0.333 \\
p_{17}=1-\frac{d_{17}}{R}=1-\frac{100 \sqrt{2}}{150}=0.057
\end{gathered}
$$

Assuming there are a source node 2 and two receivers 20 and 24. The SFA algorithm calculates the Unicast OR Shortest Distance between every pair of nodes in the network. By applying the minimum Steiner tree algorithm, we get the minimum Steiner tree connecting source node 2 with two receivers. The terminal node set is $\langle 2,20,24\rangle$. The Steiner node set is $\langle 17\rangle$. The set of links along with the OR shortest path length are: $2 \rightarrow 17(8.42209), 17 \rightarrow 20(6.51619), 17 \rightarrow$ $24(6.51619)$. The total cost of the minimum Steiner tree with OR is 21.4545 . That is the minimum expected total number of transmissions to transfer one packet successfully from node 2 to both two nodes 20 and 24 along the minimum Steiner tree. The thick red arrows in Figure 3 are the virtual links of the the minimum Steiner tree. The thin arrows in Figure 3 are the OR potential transmissions along the corresponding relay paths in the underlying wireless network.

When looking into the details of the above example on Section, we saw some disadvantages of the MSTOR algorithm. When a packet routed along the OR paths, it is required to reach specified Steiner nodes, even if it is not optimal or necessary in some cases. Let's assume one packet when transmitted from node 2 to node 17, its actual route is: $2 \rightarrow 7 \rightarrow 11 \rightarrow 16 \rightarrow 17$. Then from node 17 to node 20 , the packet follows the route $17 \rightarrow 16 \rightarrow 15 \rightarrow 20$. As a result, the packet is transmitted on link $17 \leftrightarrow 16$ twice. In addition, unicast opportunistic routing employed in MSTOR cannot fully exploit the broadcast nature of wireless medium.

In the following section, we propose a new multicast OR scheme that directly extends the OR concept to multicast to maximally take the advantages of both routing paradigms.

\section{Multicast Opportunistic Routing (MOR)}

Before getting into the details of the proposed scheme, we formally introduce several definitions and notations. 


\section{A. Definitions and notations}

- OR shortest distance between two nodes: As the result of SAF algorithm in section II-C, let $L_{S_{i} D_{j}}$ denote the OR shortest distance from transmitter node $S_{i}$ to receiver $D_{j}$ with OR routing.

- OR shortest distance between a set and a node: For a set of transmitter nodes $\mathcal{S}=\left\langle S_{1}, S_{2}, \ldots, S_{N}\right\rangle$ and a receiver $D_{j}$, we define the shortest OR distance from $\mathcal{S}$ to $D_{j}$ as the shortest OR distance from the "closest" node in $\mathcal{S}$ to $D_{j}$, i.e., $L_{\mathcal{S} D_{j}}=\min \left(L_{S_{i} D_{j}}, 1 \leqslant i \leqslant N\right)$

- Transmission Advancement: Given a transmitter node $S_{i}$, a receiver $D_{j}$, and a Candidate Forwarding Set (CFS) for $S_{i}$ to $D_{j} F_{i j}=\left\langle S_{i_{1}}, S_{i_{2}}, \ldots, S_{i_{q}}, \ldots, S_{i_{r}}\right\rangle$. For each relay node $S_{i_{q}} \in F_{i j}$, the transmission advancement $d_{i i_{q}}$ is the difference between the OR shortest distance from transmitter node $S_{i}$ to the receiver $D_{j}$ and OR shortest distance from candidate relay node $S_{i_{q}}$ to receiver $D_{j}$ :

$$
d_{i i_{q}}=L_{S_{i} D_{j}}-L_{S_{i_{q}} D_{j}}
$$

$d_{i i_{q}}$ represents the OR transmission advancement when a packet successfully transferred from transmitter node $S_{i}$ to relay node $S_{i_{q}}$ toward receiver $D_{j}$.

- Expected Transmission Advancement: In SFA algorithm, nodes in CFS $F_{i j}=\left\langle S_{i_{1}}, S_{i_{2}}, \ldots, S_{i_{q}}, \ldots, S_{i_{r}}\right\rangle$ of transmitter $S_{i}$ toward receiver $D_{j}$ always have positive transmission advancement $d_{i i_{q}}$. Assume $d_{i i_{1}} \geqslant d_{i i_{2}} \geqslant \ldots \geqslant$ $d_{i i_{q}} \geqslant \ldots \geqslant d_{i i_{r}}$. Then the OR priority order in $F_{i j}$ is $S_{i_{1}}<S_{i_{2}}<\ldots<S_{i_{q}}<\ldots<S_{i_{r}}$. The Expected Transmission Advancement (ETA) after one transmission from $S_{i}$ toward $D_{j}$ is:

$$
E T A_{i j}=\sum_{q=1}^{r} d_{i i_{q}} \cdot p_{i i_{q}} \cdot \prod_{k=0}^{q-1}\left(1-p_{i i_{k}}\right)
$$

The physical meaning of $E T A_{i j}$ is the expected number of OR transmissions achieved after one transmission from transmitter node $S_{i}$ to CFS $F_{i j}$ toward receiver $D_{j}$. The ETA metric accurately indicates the relationship between the transmission advancement and candidate selection and prioritization.

- Dedicated Transmitter Node: Given a set of transmitter nodes $\mathcal{S}=\left\langle S_{1}, S_{2}, \ldots, S_{N}\right\rangle$ and a set of receivers $\mathcal{D}$, to guarantee every node in $\mathcal{D}$ will get a copy of the packet, MOR always dedicates a transmitter $S_{i} \in \mathcal{S}$ to each receiver $D_{j} \in \mathcal{D}$. $S_{i}$ is the best candidate to forward the packet toward $D_{j}$ in order to maximize the aggregate transmission advancement from set $\mathcal{S}$ toward set $\mathcal{D}$.

\section{B. MOR Algorithm}

Now we are ready to present the MOR algorithm. MOR works in rounds. Starting with the source, MOR opportunistically employs a set of transmitters to push a packet closer to all receivers round-by-round. Each transmitter is responsible for sending the packet to a subset of receivers, and transmits the packet once each round. Based on packet receptions at the end of each round, a new transmitter set is constructed

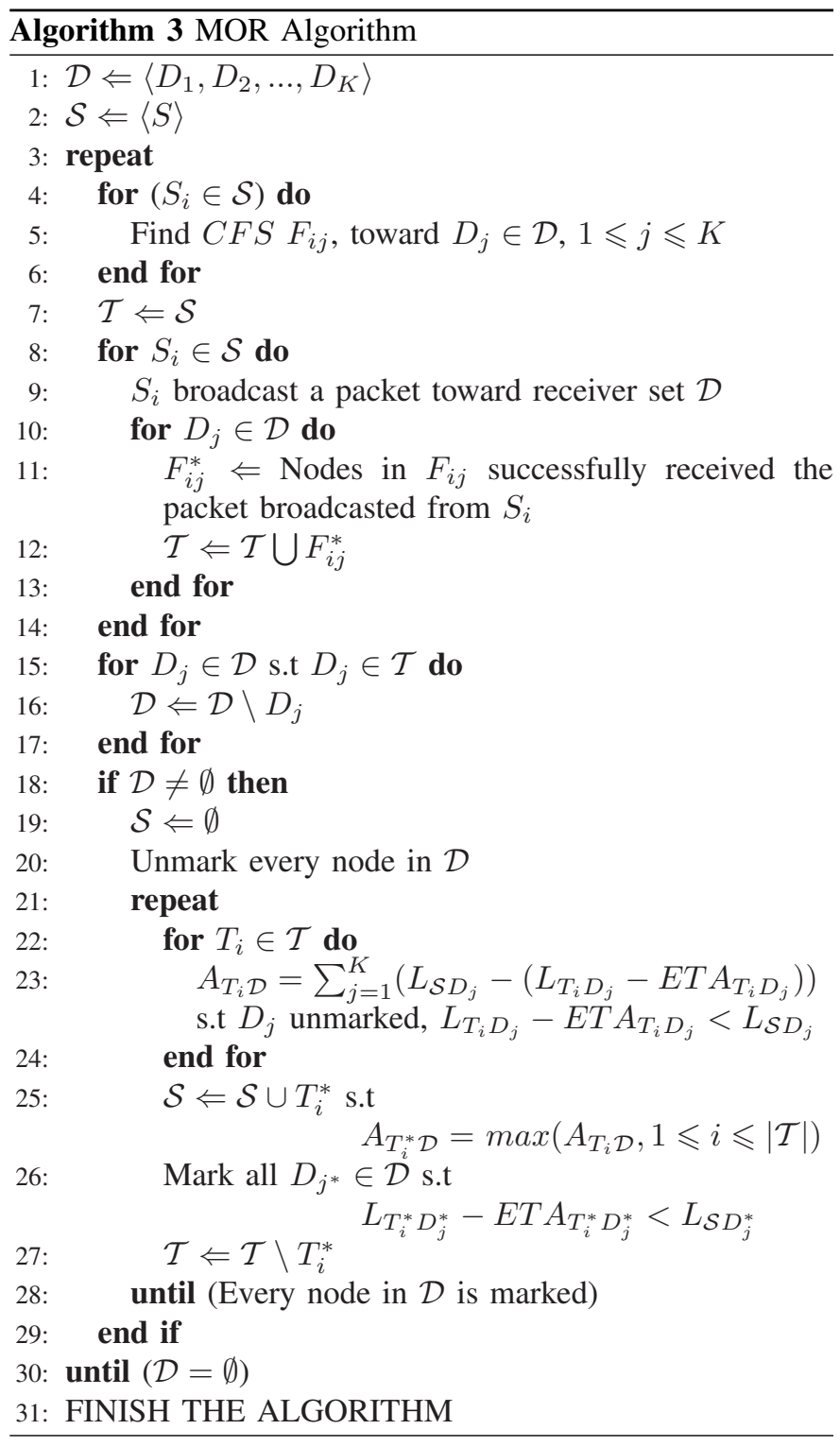

to maximize the Expected Transmission Advancement towards all receivers.

In Algorithm 3, Step 1 and Step 2 set the initial values for transmitter set $\mathcal{S}$ and receiver set $\mathcal{D}$. Notice that $\mathcal{D}$ stands for the set of receivers not yet get the packet. The Repeat-Until round between Step 3 and Step 30 runs until every receiver get the packet and $\mathcal{D}=\emptyset$. Steps 4 to 6 calculate the Candidate Forwarding Set of every transmitter node in $\mathcal{S}$ toward each receiver in $\mathcal{D}$. At Step 7, set $\mathcal{T}$ stands for the set of nodes currently have the packet. $\mathcal{T}$ initially equals to transmitter set $\mathcal{S}$. From Step 8 to Step 14, every node in the transmitter set $\mathcal{S}$ broadcasts the packet. Nodes in every CFS that successfully get the packet are added to $\mathcal{T}$. Steps 15 to 17 record the receivers just get the packet. Those receivers are removed from set $\mathcal{D}$. Step 18 checks if every receiver got the packet. If Yes, then MOR Algorithm finishes. Otherwise, it moves on to the next steps. 
Step 19 calculates the next transmitter set by initially setting $\mathcal{S}$ to an empty set. Step 20 reset dedicated transmitter nodes for all nodes in $D_{j} \in \mathcal{D}$. The Repeat-Until round between Step 21 and Step 28 runs until the algorithm assigns a dedicated relay node from $\mathcal{T}$ for each receiver in $D_{j}$. At Step 23, for each node $T_{i}$ in set $\mathcal{T}, E T A_{T_{i} D_{j}}$ stands for the Expected Transmission Advancement after one transmission of node $T_{i}$ toward receiver $D_{j}$. The condition $L_{T_{i} D_{j}}-E T A_{T_{i} D_{j}}<L_{\mathcal{S} D_{j}}$ guarantees that the expected OR shortest distance after one transmission from $T_{i}$ to $D_{j}$ is smaller than the current OR shortest distance from $\mathcal{S}$ to $D_{j}$. In other words, $T_{i}$ will bring the transmission advancement for set $\mathcal{S}$ toward $D_{j}$ if $T_{i}$ is chosen as the dedicated transmitter node for $D_{j}$ at the next round. So Step 23 calculates the Total Expected Transmission Advancement that $\mathcal{S}$ can achieve by one transmission from node $T_{i}$ toward every unmarked receiver $D_{j}$. Step 25 greedily find the relay $T_{i}^{*} \in \mathcal{T}$ to add to the transmitter set $\mathcal{S}$. $T_{i}^{*}$ is the node with the maximum total Transmission Advancement for set $\mathcal{S}$ toward receiver set $\mathcal{D}$. The following Step 26 will mark all the receivers for which $T_{i}^{*}$ is the dedicated relay node. Because $T_{i}^{*}$ is chosen as a transmitter node at the next round, Step 27 updates set $\mathcal{T}$ by removing $T_{i}^{*}$ from $\mathcal{T}$. After every receiver is assigned with a dedicated transmitter node, the new transmitter set $\mathcal{S}$ for the next round is formed. The process returns to Step 3 and repeats until every receiver got the packet.

In general, at each step, this algorithm always specify the dedicated relay node toward each receiver to maintain the reliability of the routing scheme that need to send packet to every receiver. However if the packet send out by an dedicated transmitter node toward receiver $X$ opportunistically received by a receiver $Y$ or an dedicated forwarder toward receiver $Y$, then the algorithm exploits that opportunistic reception. Said differently, the routing scheme allows opportunistic receptions across the forwarders of various receivers. The scheme also exploits the advantage of multicast transmissions at each step. That will help to significantly improve the routing performance as we will demonstrate at the following section.

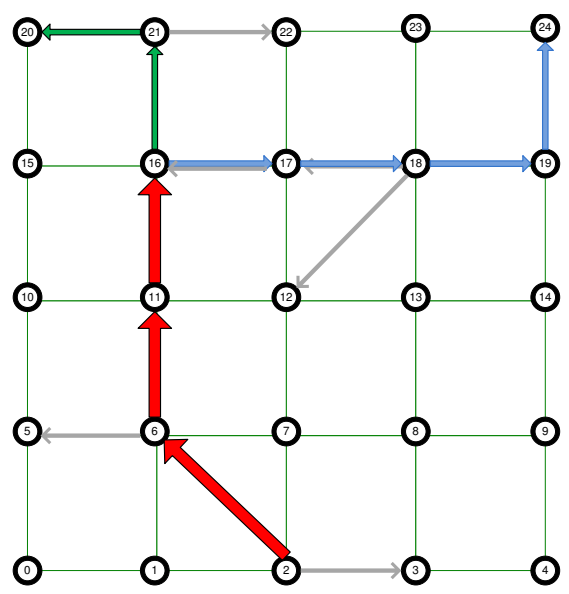

Fig. 4. Example of Multicast OR algorithm

\begin{tabular}{|c|c|c|c|}
\hline \multicolumn{4}{|c|}{ Transmission Table } \\
\hline Trans num & Transmitters & Relays to Node 20 & Relays to Node 24 \\
\hline 1 & 2 & $1(2)$ & \\
\hline 2 & 2 & $6(2)$ & $6(2)$ \\
\hline 3 & 6 & $11(6), 5(6)$ & $11(6)$ \\
\hline 4 & 11 & $16(11)$ & $16(11)$ \\
\hline 5 & 16 & & $17(16)$ \\
\hline 6,7 & 16,17 & $21(16)$ & $21(16)$ \\
\hline 8,9 & 21,17 & $16(17)$ & $18(17)$ \\
\hline 10,11 & 21,18 & $17(18), 12(18)$ & $19(18)$ \\
\hline 12,13 & 21,19 & $\mathbf{2 0}(21)$ & $22(21)$ \\
\hline 14 & 19 & & \\
\hline 15 & 19 & & $\mathbf{2 4}(19)$ \\
\hline
\end{tabular}

TABLE I

ExAmple of Multicast OR Algorithm

\section{Example of MOR and analysis}

We deploy MOR on the example presented at the Section IV-B. The performance of MOR varies, depending on the success of the actual transmission attempts. So we ran the algorithm for 1000 times and monitoring the average number of transmissions that wireless nodes made. We compare the average performance of MOR and MSTOR. The average number of transmissions to send a packet from source node 2 to receivers nodes 20 and 24 is 19.134 , which is $10 \%$ better than MSTOR $(21,45)$. Lets look into one case study with the detailed data showed in Table I. In this table, column "Relays to Node $D_{i}$ " stores the relay nodes successfully received packets, that included in the CFSs of transmitter nodes. The notation " $R_{i}\left(S_{j}\right)$ " means relay node $R_{i}$ received packet sent by sending node $S_{j}$.

The actual routes of all receivers are shown in Figure 4. MOR finds a good common path to both receivers in order to reduce the number of transmissions when the packet is still far away from the receivers. The packet does not go back and forth as MSTOR since there are no such predefined relay nodes that MOR has to send the packet to. At each round, MOR always gain the best transmission advancement as it can. Some other examples also show the ability of MOR to exploit the opportunistic receptions across the forwarders of the 2 receivers. These confirm the advantages of MOR over MSTOR.

\section{Vi. Performance evaluation}

We developed a event-driven simulator to compare the performance of Unicast OR, MSTOR, MOR and a multicast tree routing scheme presented in [4]. The multicast tree scheme is base on the EMT (Expected multicast Transmissions) metric. The physical meaning of this metric is similar to ETX but for multicast network. The EMT of a MAC layer multicast transmission is the expected number of data transmissions (including retransmissions) required for a packet to reach all the recipients. The EMT of a multicast tree is the sum over the EMT of each forwarding node. The routing scheme builds up minimum multicast tree based on EMT metric and route packets follow the tree with multicast transmissions. 


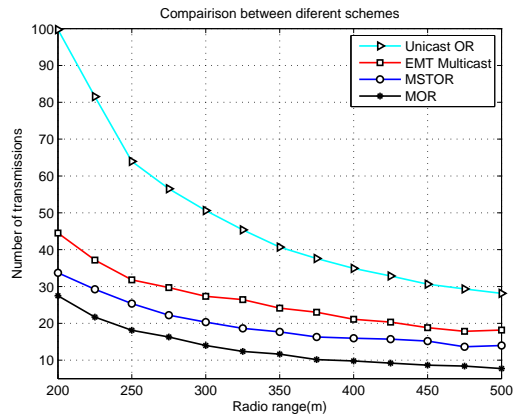

(a) Concentrated receivers

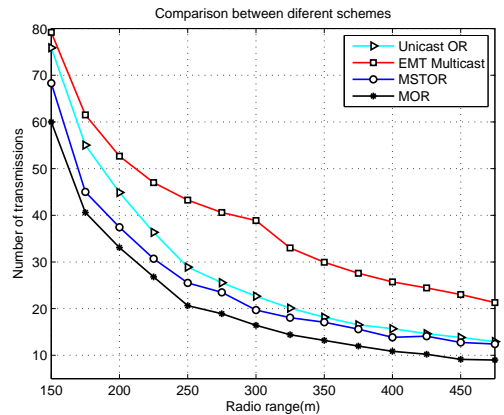

(b) Scattered receivers

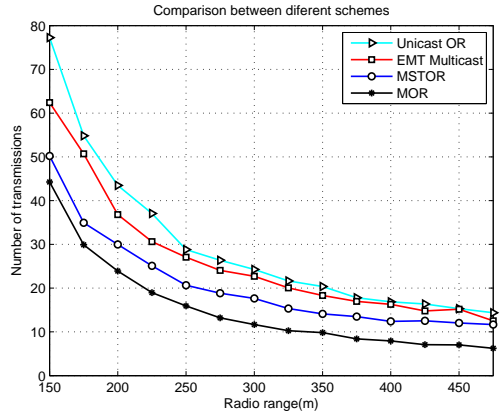

(c) Random receivers

Fig. 5. Impacts of the radio range on routing performances with different receiver distributions

The protocol was shown to have better performance than the Shortest Path Tree (SPT) and the Minimum Forwarder Tree (MFT) [4] algorithms. We choose this routing algorithm since it is the one of the best multicast-tree based wireless multicast algorithms. For convenient, we call this routing scheme is EMT Multicast.

We compare the performance of different algorithms through case studies in different network scenarios. To obtain accurate conclusions on the performance improvement of multicast OR, we systematically change several important network parameters in our case studies. For each case study, we conduct multiple simulation runs and report the average performance.

\section{A. Multicast to Concentrated receivers}

At first, we run the case study with a grid network setting. The network area is $1,000 \mathrm{~m} \times 1,000 \mathrm{~m}$. There are 100 wireless nodes evenly located in the area. Nodes are located at the crossing points of the grid. The grid size is $100 \mathrm{~m} \times 100 \mathrm{~m} .100$ nodes are numbered follow the order from the left to the right, and from the bottom to the top. Source node 0 is located at the left-bottom corner, with position $(0,0)$. The first configuration is with a set of 7 receivers, three of them are closely located at the right-top corner (Nodes 99, 89,98); 3 nodes closely located at the right-bottom corner (Nodes 9,8,19) and one node at the center (Node 55). Nodes have the same radio transmission range, and run the same routing scheme at each experiment. We gradually vary the radio transmission range from $200 \mathrm{~m}$ to $550 \mathrm{~m}$. For each setting, we run four different routing schemes multiple times and compare the average total number of transmissions.

From Figure 5(a), we see that the longer the radio range, the better the performance of all four routing schemes. With the assumption that the Packet Reception Ratio (PRR) of links are independent with each other, PRR of wireless links increases as the radio range increases. For OR, increasing radio range also expands a node's CFS. Consequently, it creates more OR paths between each pair of nodes, thus reduces the OR transmission costs. Meanwhile, the number of nodes covered by one broadcast also increases. That also improves the performance of multicast routing. Comparing

\begin{tabular}{|l|c|c|c|c|c|c|c|}
\hline \multicolumn{7}{|c|}{ Ratios between costs of other routing schemes with MOR } \\
\hline Radio range(m) & 200 & 250 & 300 & 350 & 400 & 450 & 500 \\
\hline Unicast OR & 3.63 & 3.53 & 3.62 & 3.49 & 3.56 & 3.54 & 3.65 \\
\hline EMT Multicast & 1.62 & 1.76 & 1.95 & 2.07 & 2.15 & 2.17 & 2.35 \\
\hline MSTOR & 1.23 & 1.4 & 1.45 & 1.52 & 1.63 & 1.75 & 1.81 \\
\hline
\end{tabular}

TABLE II

Performance COMPARISONS With CONCENTRATED RECEIVERS

the performance of the four routing schemes, we see that Unicast OR gets the worst performance since it does not exploit the advantages of multicast routing. With the receivers clustered together, Unicast OR is not gaining advantages of the common paths from the source node to receivers. The second worse scheme in this case is EMT Multicast. Different from Unicast OR, this routing scheme only exploits the advantage of multicast transmissions, but not OR. Our proposed routing schemes get better performance than the first two routing schemes. As expected, MOR achieves higher performance improvement than MSTOR, because it greedily exploits benefits from OR and multicast. Table II lists the ratios between the total transmission costs of other routing schemes with that of MOR at different radio ranges. MOR significantly improves the performance of both Unicast OR and EMT Multicast.

\section{B. Multicast to Scattered receivers}

We also re-run the experiments with scattered receivers. We use the same grid wireless network as the previous case study, but with only three receivers located far away from each other. The server node is located at the lower left corner with position $(0,0)$. Three receivers $(9,90,99)$ are located at the three other corners of the area. Again, we vary the radio range gradually from $150 \mathrm{~m}$ to $475 \mathrm{~m}$. For each setting, we run four different routing schemes and report the results in Figure 5(b).

From the Figure 5(b), similar to the previous experiment, the longer the radio range, the better performance for all four routing schemes. However in this case, the EMT Multicast scheme is the worst. The reason is that there is not much overlap between relay paths to the three scattered receivers. The EMT Multicast tree essentially degrades into three in- 


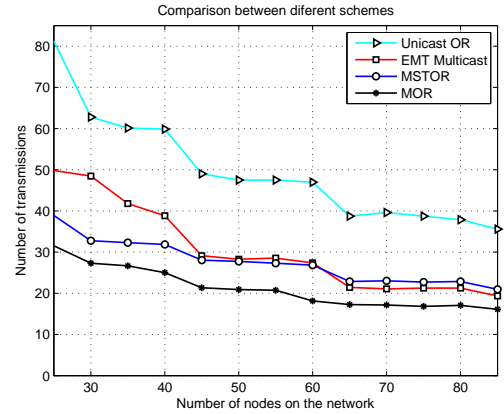

(a)

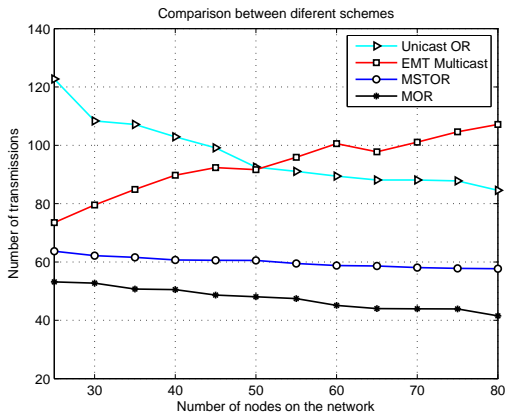

(b)

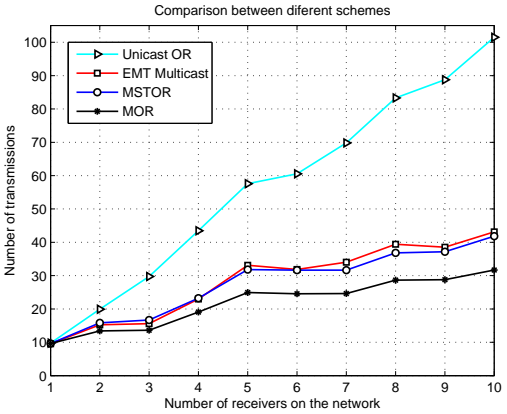

(c)

Fig. 6. Performance impact of different factors: a) the number of relay nodes under simple PRR model; b) the number of relay nodes under neighbor-dependent PRR model; c) the number of receivers.

\begin{tabular}{|l|c|c|c|c|c|c|c|}
\hline \multicolumn{7}{|c|}{ Ratios between costs of other routing schemes with MOR } \\
\hline Radio range(m) & 150 & 200 & 250 & 300 & 350 & 400 & 450 \\
\hline Unicast OR & 1.27 & 1.36 & 1.4 & 1.38 & 1.38 & 1.45 & 1.52 \\
\hline EMT Multicast & 1.32 & 1.59 & 2.1 & 2.37 & 2.27 & 2.37 & 2.52 \\
\hline MSTOR & 1.14 & 1.13 & 1.24 & 1.2 & 1.3 & 1.28 & 1.4 \\
\hline
\end{tabular}

TABLE III

PERFORMANCE COMPARISONS WITH SCATTERED RECEIVERS

\begin{tabular}{|l|c|c|c|c|c|c|c|}
\hline \multicolumn{7}{|c|}{ Ratios between costs of other routing schemes with MOR } \\
\hline Radio range(m) & 150 & 200 & 250 & 300 & 350 & 400 & 450 \\
\hline Unicast OR & 1.75 & 1.82 & 1.81 & 2.08 & 2.07 & 2.13 & 2.18 \\
\hline EMT Multicast & 1.41 & 1.54 & 1.7 & 1.95 & 1.86 & 2.05 & 2.16 \\
\hline MSTOR & 1.13 & 1.25 & 1.3 & 1.51 & 1.43 & 1.56 & 1.71 \\
\hline
\end{tabular}

TABLE IV

PERFORMANCE COMPARISONS WITH RANDOM RECEIVERS dependent paths to different receivers. Both MSTOR and Unicast OR gain performance improvements because of the advantages from Opportunistic Routing scheme. The greater radio range of nodes, the bigger performance improvement over EMT Multicast. MSTOR has slightly better performance than Unicast OR since it can exploit the advantages of the shorter routes in the minimum Steiner Tree. MOR is still the best routing scheme. It gains some multicast OR advantages when the packet is close to the source node. When the packet gets closer to the receivers, MOR only get the same advantages from opportunistic routing as Unicast OR and MSTOR. Table III is the ratios between the costs of other routing schemes with that of MOR at different radio ranges.

\section{Multicast to Random receivers}

We also run the experiments with randomly distributed receivers. We use the same grid network as the previous two cases with 6 receivers. We randomly set the positions of receiver and source nodes for 100 times. For each case, we conduct multiple simulation runs and report the average of all runs. The results are showed in Figure 5(c). Again, MOR outperforms all other schemes regardless of the positions of receiver and source nodes. Table IV is the detail of ratios between costs of other routing schemes with cost of MOR at different radio ranges.

\section{Impacts of Relay Node Density}

In this experiment, we study how relay density impacts the performance of the routing schemes. We create an random wireless adhoc network with the simple PRR model presented at Equation 3. PRR of links are independent. Wireless nodes are randomly located in an area of $1,000 \mathrm{~m} \times 1,000 \mathrm{~m}$. Nodes have homogenous radio transmission range of $200 \mathrm{~m}$. Initially, we fix one source node, 5 different receivers and 19 relay nodes. We then gradually increase the number of randomly located relay nodes from 19 to 79 and measure the number of transmissions to deliver packet successfully to every receiver. For each case, we run 1,000 simulations and report the average results in Figure 6(a). As the number of relay nodes increases, the performance of all four algorithms improves. The reason is because, as the number of relay nodes increases, the cost of the minimum Multicast tree gets smaller. The shortest OR distances between nodes are also reduced because of the expanded Candidate Forwarding Set of nodes. MOR always gets the best performance among four routing schemes regardless of the network sizes. At low relay density, MSTOR gets better performance than EMT Multicast and Unicast OR. Thanks to the combined advantages of minimum Steiner tree and OR. However, when the network is really dense, EMT Multicast could get better performance than MSTOR since multicast transmissions would achieve higher advantage than OR.

For dense network, we try a more realistic PRR model by factoring the number of neighbors around the source and destination node of a wireless link:

$$
p_{i j}=\left(1-\frac{d_{i j}}{R}\right) \frac{1}{H},
$$

where $H$ is the number of nodes who are the neighbors of node $i$, or node $j$ or both. The more neighbor nodes around, the smaller value of PRR of the link. With the same network setting as the simple PRR model, the performance results of the four routing schemes are showed on Figure 6(b). 


\begin{tabular}{|l|c|c|c|c|c|c|}
\hline \multicolumn{7}{|c|}{ Ratios between costs of other routing schemes with MOR } \\
\hline Number of receivers & 30 & 40 & 50 & 60 & 70 & 80 \\
\hline Unicast OR & 2.06 & 2.04 & 1.92 & 1.98 & 2.01 & 2.04 \\
\hline EMT Multicast & 1.51 & 1.78 & 1.91 & 2.23 & 2.3 & 2.58 \\
\hline MSTOR & 1.18 & 1.2 & 1.26 & 1.3 & 1.32 & 1.39 \\
\hline
\end{tabular}

TABLE V

PERFORMANCE COMPARISONS WITH DIFFERENT RELAY NODE DENSITIES AND MORE REALISTIC PRR MODEL

\begin{tabular}{|l|c|c|c|c|c|}
\hline \multicolumn{6}{|c|}{ Ratios between costs of other routing schemes with MOR } \\
\hline Number of receivers & 2 & 4 & 6 & 8 & 10 \\
\hline Unicast OR & 1.48 & 2.28 & 2.47 & 2.91 & 3.2 \\
\hline EMT Multicast & 1.14 & 1.21 & 1.3 & 1.38 & 1.36 \\
\hline MSTOR & 1.18 & 1.22 & 1.29 & 1.29 & 1.32 \\
\hline
\end{tabular}

TABLE VI

PERFORMANCE COMPARISONS WITH DIFFERENT NUMBER OF RECEIVERS

As the number of relay nodes increases, the performance of EMT Multicast gets worse. The introduction of a new node decreases the PRRs on many nearby links. If some links are included in the EMT Multicast tree, it will increase the multicast transmission costs. However, the performance of Unicast OR, MSTOR and MOR improve as the number of relay nodes in the network increases. With a high node density, OR can put more candidate relays into the forwarding sets. This compensates the negative impact from the reduced PRRs on each individual links. Among the four, MOR still has the best performance. Table $\mathrm{V}$ presents the ratios between costs of other routing schemes with the cost of MOR with different relay node densities and more realistic PRR model.

\section{E. Impacts of the Number of receivers}

Lastly, we study the performance of different routing schemes with different numbers of receivers. With the same grid network at the Section VI-D, we fixed the network size and set up 45 randomly located wireless nodes. We then randomly choose and increase the number of receivers from one to ten. We run each routing scheme 1,000 times and calculate the average number of transmissions made in each case. The results are showed in the Figure 6(c). MOR still gets the best performance compared with the other routing schemes. MSTOR and EMT Multicast have almost the same performance. As the number of receivers increases, the cost of Unicast OR increases almost linearly. That is because Unicast OR schemse was designed for Unicast fraffic. Table VI presents the ratios between costs of other routing schemes with the cost of MOR at different numbers of receivers.

\section{CONCLUSION}

In this paper, we investigated the gain of adopting Opportunistic Routing (OR) in wireless multicast. We proposed two schemes to simultaneously take the advantages of OR and multicast. The first scheme, MSTOR, combines Minimum Steiner Tree with Unicast OR. Using the shortest OR distances between nodes as virtual link weights, we constructed virtual minimum Steiner tree and mapped it into Unicast OR relay paths connecting the source with all receivers. To fully exploit opportunistic receptions, we extended the OR concept to directly meet the multicast needs. The proposed MOR algorithm opportunistically updates transmitter set to maximally push the packet to all receivers. Through numerical simulations, we showed that both MSTOR and MOR outperform Unicast OR and existing multicast tree based algorithms. MOR achieves significant transmission cost saving in all studied cases.

Our current evaluations assume a simplified packet loss model. As a work for future, we will evaluate the performance of MSTOR and MOR with more realistic loss models. Based on existing implementation of Unicast OR, the proposed MSTOR algorithm can be readily implemented and tested using packet-level simulators, such as OPNET. The implementation of MOR is less straightforward. To fully explore the gain of OR in multicast, the current MOR algorithm incurs significant signaling and synchronization overhead. As a future work, we will tailor the MOR algorithm for practical implementation. In the process, we will further investigate the trade-off between the complexity and performance gain of OR in wireless multicast.

\section{REFERENCES}

[1] W. S. Sung-Ju Lee and M. Gerla, "On-demand multicast routing protocol in multihop wireless mobile networks," vol. 7, no. 6. Springer, 2002, pp. 441-453.

[2] J. Wieselthier, G. Nguyen, and A. Ephremides, "On the construction of energy-efficient broadcast and multicasttrees in wireless networks," in IEEE Infocom 2000, Tel Aviv, Israel, 2000.

[3] V. Raghunathan, V. Borkar, C. Min, and P. Kumar, "Index Policies for Real-Time Multicast Scheduling for Wireless Broadcast Systems," in IEEE Infocom 2008, Phoenix, AZ, 2008.

[4] X. Zhao, C. T. Chou, J. Guo, and S. Jha, "Probabilistically reliable on-demand multicast in wireless mesh networks," in International Symposium on a World of Wireless, 2008.

[5] S. Shakkottai, X. Liu, and R. Srikant, "The multicast capacity of large multihop wireless networks," in MobiHoc '07: Proceedings of the 8th ACM international symposium on Mobile ad hoc networking and computing. New York, NY, USA: ACM, 2007, pp. 247-255.

[6] S. Biswas and R. Morris, "Opportunistic routing in multi-hop wireless networks," in In Proceedings of the Second Workshop on Hot Topics in Networks (HotNets-II), Cambridge, MA, Nov. 2003.

[7] H. Dubois-Ferrire, M.Grossglauser, and M. Vetterli, "Least-cost opportunistic routing," in 2007 Allerton Conference on Communication, Control, and Computing, Monticello IL, September 2007.

[8] R.C.Shah, S.Wietholter, A.Wolisz, and J.M.Rabaey, "When does opportunistic routing make sense ?" in in IEEE PerSens, Mar.2005.

[9] K. Zeng, W. Lou, and H. Zhai, "On End-to-end Throughput of Opportunistic Routing in Multirate and Multihop Wireless Networks," in IEEE Infocom 2008, Phoenix, AZ, April 15-17, 2008.

[10] T.Le and Y.Liu, "On the Capacity of Hybrid Wireless Networks with Opportunistic Routing," in WASA'09, Boston, USA, August 2009.

[11] S. Chachulski, M. Jennings, S. Katti, and D. Katabi, "Trading structure for randomness in wireless opportunistic routing," in In Proc. ACM Sigcomm, 2007.

[12] R. Laufer, H. Dubois-Ferrire, and L. Kleinrock, "Multirate Anypath Routing in Wireless Mesh Networks," in IEEE Infocom 2009, Rio de Janeiro, Brazil, April 2009.

[13] P. Ruiz and A. Gomez-Skarmeta, "Approximating Optimal Multicast Trees in Wireless Multihop Networks," in Computers and Communications, 2005. ISCC 2005. Proceedings. 10th IEEE Symposium on, Cartagena, Spain, 27-30 June 2005, pp. 686-691.

[14] S. E. Dreyfus and R. A. Wagner, "The Steiner problem in graphs," in Networks, 1972, pp. 195-207. 